\title{
IV. JOINT DISCUSSION ON ASTRONOMICAL OBSERVATIONS WITH ARTIFICIAL SATELLITES, ROCKETS AND BALLOONS \\ (I3 August 1958)
}

Organizing Committee: Prof. F. L. Whipple (Chairman), Dr A. Dollfus and Dr O. A. Melnikov.

Chairman: Prof. F. L. Whipple.

For a number of years sounding rockets have been sent to great altitudes in the Earth's atmosphere to measure geophysical quantities of prime importance. Since there is no clear-cut dividing line between geophysics and astronomy, a great deal of astronomical understanding has been gleaned from this high-altitude rocket research. Of particular interest has been the measurement of solar radiation in the far ultra-violet beyond the limits of transparency in the Earth's atmosphere and, indeed, to the X-ray region of a few angstrom units. Now the rocket technique has been extended to measurements in the region of Lyman-alpha at great distances in space.

The potential of the balloon as a carrier of astronomical equipment above the major absorbing regions of the Earth's atmosphere has been little utilized. We shall hear some reports of new results with this old but greatly improved vehicle.

The recent successes by the U.S.S.R. and the U.S.A. in launching artificial Earth satellites during the International Geophysical Year have opened a new and striking panorama for future research in astronomy.

It is highly fitting, therefore, that a Joint Discussion should be held on the current status of research results from these new and powerful tools of astronomy. The day has now passed when the astronomer need limit himself to observations constrained and distorted by the opacity, irregularities and turbulence of our atmosphere. He can now send his measuring instruments into open space and analyse all of the radiations and particles that reach our local volume of space.

During this session, which was organized by a committee comprising A. Dollfus of France, O. A. Melnikov of the U.S.S.R. and your chairman, we shall hear of striking results obtained by early applications of these new and powerful methods.

FRED L. WHIPPLE

\section{r. OBSERVATIONS ASTRONOMIQUES A BORD DE BALLONS LIBRES: ETUDE DE LA STRUCTURE FINE DE LA GRANULATION SOLAIRE}

AUDOUIN DOLLFUS

L'idée d'utiliser le ballon libre pour améliorer certaines observations astronomiques a été appliqué pour la première fois à la fin du siècle dernier, par l'astronome Janssen, fondateur de l'Observatoire de Meudon. Janssen fit effectuer des mesures spectrales à différentes altitudes, afin de discriminer les raies atmosphériques des raies photosphériques dans le spectre du Soleil. Au début de notre siècle, d'autres ascensions analogues ont été organisées pour dénombrer les étoiles filantes lors des pluies de Léonides. Une astronome américaine, Miss Klumpke, et un astronome russe, le Pr. Tikhoff, prirent part, à Paris, à certains de ces vols.

Ensuite, plus de 50 années se sont écoulées sans que cette méthode d'observation fut reprise. Il semble que ce soit un cas rare dans l'histoire de l'Astronomie qu'un procédé d'observation si fructueux ait été abandonné si longtemps.

En I952, j'ai entrepris un nouveau programme: Un aérostat de $\mathrm{I} 200 \mathrm{~m}^{3}$ a été spécialement étudié pour ce but. L'étoffe en soie caoutchoutée, très légère, pesait seulement r4o Kg. La nacelle, en osier flexible, portait un dispositif d'oxygénation pour la respiration à grande altitude, et des organes de vol, tous largables par parachutes. Un personnel technique a été formé et entraîné pour la manipulation de ce matériel. 


\section{JOINT DISCUSSION}

Pour étudier les meilleures conditions de l'emploi de la nacelle d'un aérostat comme support d'un télescope astronomique, des ascensions préliminaires ont été effectuées en r952 et I953, à différentes altitudes.

Le 30 Mai I954 un vol a été entrepris, de nuit, jusqu'à $7000 \mathrm{~m}$. Ce vol était destiné d'une part à étudier les conditions d'emploi d'un télescope de $30 \mathrm{~cm}$ de diamètre, d'autre part à doser la teneur en vapeur d'eau dans la haute atmosphère terrestre. La lumière recueillie par des miroirs traversait un filtre polarisant isolant la bande atmosphérique $8300 \AA$ de la vapeur d'eau, puis un dispositif balayant cette portion du spectre. Les cellules photoélectriques donnaient de la sorte un courant modulé, proportionnellement à l'intensité de la bande spectrale [r]. Le ballon était gonflé au départ à la moitié seulement de son volume avec de l'hydrogène. Il était piloté par Charles Dollfus. Le vol a montré que la quantité d'eau restant dans l'atmosphère au-dessus de $7000 \mathrm{~m}$ représentait le centième de la quantité d'eau totale contenue dans l'atmosphère. Cette expérience a également montré que la stabilité d'un aérostat était suffisante pour permettre la réalisation de certaines observations astronomiques.

Deux nouveaux programmes ont alors été établis. Le premier concerne la recherche de la vapeur d'eau dans l'atmosphère de la planète Mars. La technique aérostatique, nouvelle, consiste en l'emploi d'un grand nombre de ballons dilatables accrochés le long d'un cable vertical. La cabine étanche est surmontée d'un télescope de $50 \mathrm{~cm}$ de diamètre. L'observateur pourra atteindre une altitude de $20000 \mathrm{~m}$ au moins. La préparation de l'expérience n'est pas encore achevée.

Le second programme, maintenant complétement réalisé, concerne l'observation de la structure fine de la granulation de la photosphère solaire. Dans les observations du Soleil, le pouvoir grossissant des télescopes ordinaires est limité par les imperfections de l'atmosphère terrestre, échauffée par le rayonnement solaire. Le pouvoir de résolution des meilleures images de la photosphère obtenues à ce jour ne dépasse guère 0 " 70 et correspond à la puissance d'un objectif de $18 \mathrm{~cm}$ seulement. Un télescope emmené à bord d'un ballon est entraîné avec l'air, il domine par son altitude les zones les plus troublées de l'atmosphère et il travaille dans un milieu dont l'indice de réfraction est plus voisin de l'unité. Le programme a été réalisé en collaboration avec l'Observatoire de Cambridge, Angleterre.

Le télescope a été construit à Cambridge par les Dr Blackwell et Dewhirst. Il comprenait un objectif de $30 \mathrm{~cm}$, un tube de $5 \mathrm{~m}$ de long en charpente métallique légère, une optique auxiliaire et une caméra munie d'un obturateur rapide exposant une fraction de millisecondes. L'image du Soleil mesurait $45 \mathrm{~cm}$ de diamètre; l'émulsion était un film microfile Kodak à très grande résolution [2].

Le matériel aérostatique a été adapté à l'Observatoire de Meudon, où le télescope a été transporté pour être fixé sous la nacelle.

Deux vols ont été effectués, autour de $6000 \mathrm{~m}$, l'un le 22 Novembre I956, l'autre le I Avril I957. Le ballon était piloté par l'auteur accompagné du Dr Blackwell; environ 400 photographies ont été réalisées au cours de chacun des deux vols.

Les images obtenues sont très satisfaisantes; leur résolution approche celle correspondant à la puissance maximum de l'objectif et elles montrent des détails nettement plus fins que ceux obtenus au préalable au niveau du sol.

L'examen des clichés confirme le caractère nettement individuel de chaque granule. Leurs dimensions moyennes sont environ $I^{\prime \prime} 5$. Très peu de spécimens sont plus grande que $2^{\prime \prime}$, peu plus petits que 0.8 . Cependant, vu individuellement, chaque grain présente une structure beaucoup plus variée que nous ne le supposions au préalable. Ils sont rarement circulaires; leur forme est souvent polygonale, allongée, arguée ou déchiquetée. Ils ont souvent une brillance uniforme, avec des bords nets; cependant certains sont filandreux ou même très flous; certains sont aussi constitués par l'agglomération de petites taches, en bouquets. Leur contraste est inégal. On observe des croissants, des couronnes, mais ces figures complexes s'expliquent par l'association de plusieurs grains. Entre les granules, le matière photosphérique moins brillante n'est point uniforme. Il y a des lacunes; certains grains semblent manquer, leur emplacement étant marqué par une tache, généralement plus sombre que la matière intergranulaire voisine. 


\section{SATELLITES, ROCKETS, BALLOONS}

Les clichés montrent également des structures intéressants des étendues plus vastes. Quelquefois de singuliers alignements courent, isolés ou parallèles, comme des stries ou des coups de rateaux; ils concernent, soit les granules eux-mêmes, soit la matière sombre. En plusieurs endroits, aussi, des régions quelquefois aussi grandes que $30^{\prime \prime}$ de diamètre se montrent parsemées de grains plus petits, et un peu plus serrés. Nous savions déjà, depuis les travaux de Lyot, que les granules sont souvent plus petits autour de la pénombre des taches, là où les champs magnétiques sont plus intenses.

Les clichés pris au bord du disque nous montrent les granules jusque près du contour du Soleil. Leur contraste devient très faible à $40^{\prime \prime}$ du bord, la profondeur optique concernée y étant voisine de la surface de transition avec la chromosphère. Les granules sont plus grossières et allongés parallèlement au bord. Cette propriété, que Lyot avait déjà signalée, en I943, a été clairement confirmée par J. Rösch plus récemment; elle prouve que les masses brillantes sont des colonnes verticales dont la hauteur est limitée. Les images obtenues en ballon au bord du disque, moins réussies que celles des parties centrales, confirment les observations antérieures, mais sans apporter, sur ce point, nettement plus de détails.

Des courbes d'égale intensité ont été tracées, pour différentes valeurs de la brillance, à l'aide du traceur d'isophotes de l'Observatoire Sacramento Peak dans le New Mexico. Les lignes isophotes montrent clairement la grande complexité de la forme des granules. Grâce à la qualité de l'image dépourvue de la diffusion par les troubles atmosphériques, le contraste entre les granules brillants et les intervalles sombres peut être déterminé sans trop d'altération. Les mesures, corrigés des effets instrumentaux, donnent 30 à $40 \%$.

La courbe d'auto-corrélation qui lie les écarts de brillance entre de nombreux points choisis à des distances croissantes les unes des autres a été établie avec l'aide du calculateur électronique de l'Université de Cambridge. La partie initiale de la courbe montre une branche descendante dont la demi-largeur est $0^{\prime \prime} 57$. Cette valeur fixe l'ordre de grandeur de la résolution; elle permet de comparer la qualité de différents clichés. Les photographies du Dr Miller, obtenues au niveau du sol, étudiées par G. Wlérick, donnaient o"9. Le gain de pouvoir résolvant est donc très appréciable.

L'étude complète de clichés, entreprise par les Dr Blackwell, Dewhirst et l'auteur, n'est pas encore achevée.

\section{RÉFÉRENCES}

[I] Dollfus, A. C.R. 239, 954, 1954 .

[2] Blackwell, D., Dewhirst, D. and Dollfus, A. Nature, Lond. r8o, 2 I r, 1957.

\section{SOLAR GRANULATION PHOTOGRAPHS FROM THE STRATOSPHERE}

\section{MARTIN SCHWARZSCHILD}

The Princeton University Observatory carried out last summer three unmanned stratospheric balloon flights for the same purpose as that described in the preceding communication, namely to obtain high-definition photographs of the solar granulation. The special telescope for this purpose was built by the Perkin-Elmer Corporation, the electronic pointing mechanism was constructed by the Boulder Research Laboratories, and the balloons were manufactured and flown by General Mills Inc. The project was sponsored by the U.S. Office of Naval Research and by the U.S. Air Force.

The telescope consisted of a fused quartz mirror with an aperture of 12 in. and a focal length of $8 \mathrm{ft}$. The primary image was enlarged twenty-five times through a small lens, so that the effective focal length was $200 \mathrm{ft}$. The photographs were taken on $35-\mathrm{mm}$ finegrain film $\left(\lambda \lambda_{5}\right.$ I00 $\AA$ and $\left.5900 \AA\right)$ with one frame each second and with an exposure time of $I / I 000$ of a second and a frame size of $I \cdot 2$ by $I \cdot 7$ minutes of arc. Care was taken to minimize thermal disturbances within the instrument; in particular, the small secondary 